\section{Antropometria de escolares ao ingresso no ensino fundamental na cidade de Belém, Pará, 2001}

\section{School aged anthropometry when enrolling in the first grade of elementary school in the city of Belém, Pará, 2001}

Olga Maria Domingues das Neves 1 Anne Lise Dias Brasil 2 Laélia Maria Barra Feio Brasil 3 José Augusto de Aguiar Carrazedo Taddei 4

1,3 Disciplina Pediatria. Departamento de Assistência Materno Infantil II. Universidade Federal do Pará. Belém, PA, Brasil 2,4 Disciplina de Nutrologia. Departamento de Pediatria Universidade Federal de São Paulo. Rua Loefgreen, 1647. São Paulo, SP, Brasil. CEP: 04.040-032. E-mail: nutmet@terra.com.br

\begin{abstract}
Objectives: to perform anthropometric measurements in elementary school students of the public school system of the State of Belém, Pará, as a tool for nutritional surveillance and to study associations between nutritional status and biological, social and economic characteristics.

Methods: 793 students were assessed for their nutritional status, by $z$ scores of height/age and weight /height according to the World Health Organization criteria. Biological, social and economic variables were: gender, age, place of birth, age of school enrolment and maternal education level. The chi square test was used to relate nutritional status to variables.

Results: of the 637 students assessed, 16.6\% were of low stature or in risk of low stature, $4.5 \%$ malnourished or in risk of malnourishment, $7.4 \%$ were overweight or obese. There was no significant association between nutritional status and gender. The majority of the children was born in the capital, was enrolled late in school and had mothers with education level of $\geq 4$ years. There was a significant correlation between obesity and low stature with low maternal education level; malnutrition risk and low stature with late school enrolment; and obesity and normal stature with regular enrollment.

Conclusions: low maternal school level and late school enrollment are associated to nutritional problems. School nutritional surveillance is important to assess, treat and prevent children's health and nutrition risks.
\end{abstract}

Key words Anthropometry, Nutritional surveillance, Nutrition assessment

\section{Resumo}

Objetivos: realizar a antropometria em escolares da primeira série da rede pública estadual em Belém, Pará, como instrumento de vigilância nutricional e estudar as associações entre o estado nutricional e características biológicas e socioeconômicas.

Métodos: foram avaliados nutricionalmente 793 escolares, pelos escores $z$ de estatura/idade $e$ pesolestatura, segundo a Organização Mundial da Saúde. As variáveis biológicas e socioeconômicas foram: sexo, idade, naturalidade, idade de ingresso da criança na escola e escolaridade materna. Utilizou-se o teste quiquadrado ao relacionar o estado nutricional com as variáveis.

Resultados: em 637 escolares observamos 16,6\% de baixa estatura e risco de baixa estatura, 4,5\% de desnutrição e risco de desnutrição, 7,4\% de sobrepeso e obesidade. Não houve associação significante entre estado nutricional e sexo. A maioria das crianças nasceu na capital, ingressou tardiamente na escola e tinham mães com escolaridade $\geq 4$ anos. Observou-se correlação significante entre obesidade e baixa estatura com menor escolaridade materna; risco de desnutrição e baixa estatura com o ingresso escolar tardio; e obesidade e estatura normal com o ingresso regular.

Conclusões: a menor escolaridade materna e o ingresso tardio escolar estão associados à presença de desvios nutricionais. A vigilância nutricional escolar é importante para avaliar, tratar e prevenir os riscos para saúde e nutrição infantil.

Palavras-chave Antropometria, Vigilância nutricional, Avaliação nutricional 


\section{Introdução}

As crianças têm padrões de crescimento semelhantes, apesar das mais variadas origens étnicas e geográficas. As diferenças no crescimento estão mais ligadas aos fatores ambientais e socioeconômicos do que aos de origem genético-racial. ${ }^{1}$ Os fatores ambientais, como a nutrição adequada, são verdadeiros moduladores do fenótipo. Aos seis e sete anos de idade a estatura reflete os fatores socioeconômicos e ambientais em que vive a criança. ${ }^{2}$

A estatura é a medida antropométrica que melhor reflete a condição socioeconômica, sanitária e cultural de uma população, sendo a mesma usada como instrumento de vigilância nutricional. ${ }^{2}$

Bengoa propôs a medida de estatura da criança ao ingresso na escola primária como indicador para ilustrar a história passada de nutrição de uma comunidade, a qual está estreitamente relacionada com o nível de desenvolvimento da população. ${ }^{3}$

A avaliação nutricional de crianças, por meio da vigilância nutricional, utilizando-se repetidas coletas de dados antropométricos, permite orientar o planejamento, execução e avaliação de programas de saúde em uma determinada população. 4

No continente americano, a avaliação nutricional dos escolares de primeiro grau, através do censo de estatura, iniciou-se na Costa Rica, em 1979, ligada à decisão política de identificar a população em alto risco de desnutrição. Estes grupos seriam sujeitos à intervenção com programas e projetos orientados a modificar os fatores causais do problema nutricional detectado. Nos países da América Central ainda é usado esse procedimento valioso e de baixo custo para detectar comunidades que têm os mais altos níveis de desnutrição. ${ }^{3}$

Monteiro 4 propôs, em 1989, o estabelecimento de um sistema nacional de monitorização do crescimento infantil no Brasil, viabilizado com a coleta anual da estatura de todos os alunos ingressantes nas escolas de primeiro grau do país. A curto prazo, a implantação da proposta permitiria identificar as regiões e microrregiões que deveriam ser priorizadas pelos programas de desenvolvimento. A médio prazo, as informações geradas auxiliariam na avaliação do grau de sucesso das políticas nacionais e regionais de desenvolvimento. Foram realizados alguns censos de estatura de escolares ingressantes na primeira série do primeiro grau em estados do Nordeste, como Paraíba, Ceará e Piauí e em algumas cidades do Sudeste e Sul como Osasco, São Paulo e Florianópolis, Santa Catarina, mas apenas como pesquisas pontuais e não como rotina de vigilância de crescimento. $5-7$
Este trabalho tem como finalidade avaliar o estado nutricional de crianças ingressantes no ensino fundamental na cidade de Belém, Pará, bem como as associações com variáveis biológicas e socioeconômicas.

\section{Métodos}

Foi realizado um estudo transversal como instrumento de vigilância nutricional em escolares da primeira série da rede pública estadual, ao ingresso no ensino fundamental, na cidade de Belém, Pará, localizada na região Amazônica, região Norte do Brasil, no período de outubro a novembro de 2001, por meio da antropometria (peso e estatura) e de um questionário com enfoque em fatores biológicos e socioeconômicos.

A amostra foi obtida a partir da divisão da população de estudo em grupos de unidades . As unidades no primeiro estágio foram representadas pelos bairros, sendo sorteados dois bairros em cada um dos quatro distritos administrativos de maior densidade demográfica da cidade. Foi considerado o primeiro bairro sorteado em cada distrito, deixando o segundo como reserva, caso fosse impossível obter o número mínimo de crianças no primeiro. A seguir foi realizado o sorteio das escolas da rede pública estadual nos bairros sorteados. Foram sorteadas duas escolas para cada bairro com o mesmo critério adotado no primeiro estágio.

Para o cálculo do tamanho amostral utilizou-se a sub-rotina Statcalc do programa estatístico Epi-info, 6.0. Considerou-se uma população aproximada de 10.000 novos escolares matriculados no ano de 2001, na primeira série e com a estimativa de baixa estatura em $10 \%$, para um erro amostral da ordem de $20 \%$ e intervalo de confiança de $95 \%$. A antropometria foi, então, realizada em 793 alunos. Os critérios de exclusão observados foram: presença de malformações congênitas ou adquiridas, doenças crônicas e deficiência física ou mental.

As medidas de peso e estatura dos escolares foram realizadas pela pesquisadora e por dois discentes de Medicina devidamente treinados quanto à padronização de técnicas. As medidas foram realizadas por duas vezes seguidas e calculada a sua média aritmética, sendo anotado o resultado em formulário padronizado.

$\mathrm{O}$ equipamento antropométrico consistiu de balança portátil digital da marca Kratos $®$, com capacidade de $150 \mathrm{~kg}$ e precisão de $50 \mathrm{~g}$, colocada sobre superfície plana. As crianças foram pesadas usando apenas roupa íntima e sem calçados. Para 
isso permaneciam eretas no centro da balança, com os braços esticados ao lado do corpo, sem se movimentarem. ${ }^{8}$ A variação admitida entre as duas medidas de peso foi de no máximo $50 \mathrm{~g}$.

Para aferição da estatura foram utilizados fita métrica plástica inextensível fixada verticalmente, com fita adesiva em parede lisa, e um esquadro de madeira. Uma plataforma de madeira foi usada para evitar o desnível do rodapé. A criança foi posicionada de costas e de pé, sem sapatos e sem adereços nos cabelos, com os pés unidos, encostados na parede, o mesmo ocorrendo com a região glútea, ombros e o ponto mais protuberante da região parieto occiptal. Com a mão direita o pesquisador manteve apoiado o esquadro de madeira sobre a cabeça da criança, em ângulo reto com a parede. Com a mão esquerda, ele apoiava a mandíbula da criança, para retificar a cabeça. A variação admitida entre as duas medidas de estatura foi de, no máximo, $0,4 \mathrm{~cm}$.

A estatura para a idade (E/I) o peso para a estatura $(\mathrm{P} / \mathrm{E})$ foram os indicadores antropométricos utilizados. O estado nutricional foi avaliado a partir do cálculo de escores $z$ dos índices acima descritos para as médias e desvios-padrão da população de referência National Center of Health Statistics (NCHS). 9 Para a classificação nutricional foram utilizados os pontos de corte descritos nos Quadros 1 e 2.8

As variáveis biológicas e socioeconômicas estu-

\section{Quadro 1}

Classificação do estado nutricional pelo indicador peso/estatura (P/E).

\begin{tabular}{|ll|}
\hline Grupos & Escores $z$ \\
\hline Desnutrição atual & $<-2,0$ \\
Risco de desnutrição & $\geq-2,0 \mathrm{a} \leq-1,5$ \\
Eutrofia & $>-1,5 \mathrm{a}<+1,5$ \\
Sobrepeso & $\geq+1,5 \mathrm{a} \leq+2,0$ \\
Obesidade & $>+2,0$ \\
\hline
\end{tabular}

\section{Quadro 2}

Classificação do estado nutricional pelo indicador estatura/ idade (E/I).

\begin{tabular}{|ll|}
\hline Grupos & Escores $z$ \\
\hline Baixa estatura (BE) & $<-2,0$ \\
Risco de baixa estatura (Risco BE) & $\geq-2,0$ a $\leq-1,5$ \\
Estatura normal & $>-1,5$ \\
\hline
\end{tabular}

dadas foram: sexo, idade, naturalidade, escolaridade materna e idade de ingresso na escola. Foi considerado como regular o ingresso na escola em idade inferior a 96 meses e após esse período foi considerado como tardio. Os dados relativos a sexo, data de nascimento e procedência da criança foram obtidos nas fichas dos alunos, disponíveis nas secretarias das escolas. O nível de escolaridade da mãe foi obtido através de informação da criança, sendo complementada pela própria mãe, nos casos em que o aluno não sabia informar.

O banco de dados foi analisado nos programas Epi-info e Epinut utilizando tabelas de contingência para a descrição das frequiências e estudo de associações entre as variáveis.

Foram excluídos da análise estatística 156 escolares $(19,6 \%)$ com mais de 120 meses, idade a partir da qual se torna imprecisa a análise pelo Epinut, restando 637 crianças para as análises. Na análise do índice peso/estatura foram excluídas pelo mesmo programa mais quatro crianças do sexo feminino, com estatura superior a $137 \mathrm{~cm}$.

Utilizou-se o teste Qui-quadrado $\left(\chi^{2}\right)$, com o nível de significância de 0,05 , para avaliar a relação do estado nutricional do escolar com as outras variáveis estudadas. 10

\section{Resultados}

Dos 637 escolares, $359(56,4 \%)$ eram do sexo masculino e 278 do sexo feminino (43,6\%); a idade variou de 72 a 113 meses, sendo a maior frequiência na faixa de $\geq 84$ a $<108$ meses. Quanto à naturalidade, $88,1 \%$ dos escolares nasceram na cidade de Belém, 10,4\% eram naturais de outros municípios do interior do Estado do Pará e apenas 1,6\% eram procedentes de outros estados do país. Observou-se que $75,8 \%$ das mães terminaram os quatro primeiros anos do ensino fundamental.

A análise do escore $z$ de $\mathrm{P} / \mathrm{E}$ revelou a prevalência de 0,9\% de desnutrição (IC95\%: 0,4-2,2). Em risco de desnutrição foram encontrados $3,6 \%$ das crianças, $88 \%$ eram eutróficas e $3 \%$ apresentavam sobrepeso. O percentual de obesidade foi de $4,4 \%$. Nenhuma criança se encontrava abaixo do escores $z$ 3,0 .

Comparando a naturalidade da criança com a escolaridade materna, observou-se significância estatística na relação entre a menor escolaridade materna ( $<4$ anos) e a procedência de outros municípios e estados.

Em relação à idade de ingresso na escola, observou-se que 302 crianças $(47,4 \%)$ tiveram idade 
Distribuição dos escolares de primeira série de escolas públicas estaduais, segundo o estado nutricional e a escolaridade materna. Belém, Pará, 2001.

\begin{tabular}{|c|c|c|c|c|c|c|}
\hline & \multicolumn{2}{|c|}{$\geq 4$ anos } & \multicolumn{2}{|c|}{$<4$ anos } & \multicolumn{2}{|c|}{ Total } \\
\hline & $\mathrm{n}$ & $\%$ & $n$ & $\%$ & $\mathrm{n}$ & $\%$ \\
\hline Eutrofia & 423 & 87,8 & 134 & 88,7 & 557 & 88,0 \\
\hline Sobrepeso & 17 & 3,5 & 2 & 1,3 & 19 & 3,0 \\
\hline Obesidade & 17 & 3,5 & 11 & 7,3 & 28 & 4,4 \\
\hline Total & 482 & 76,1 & 151 & 23,9 & 633 & 100,0 \\
\hline
\end{tabular}

a $\chi^{2}=3,84 ; p=0,050$

Tabela 2

Distribuição dos escolares de escolares de primeira série de escolas públicas estaduais, segundo o estado nutricional e a escolaridade materna. Belém, Pará, 2001.

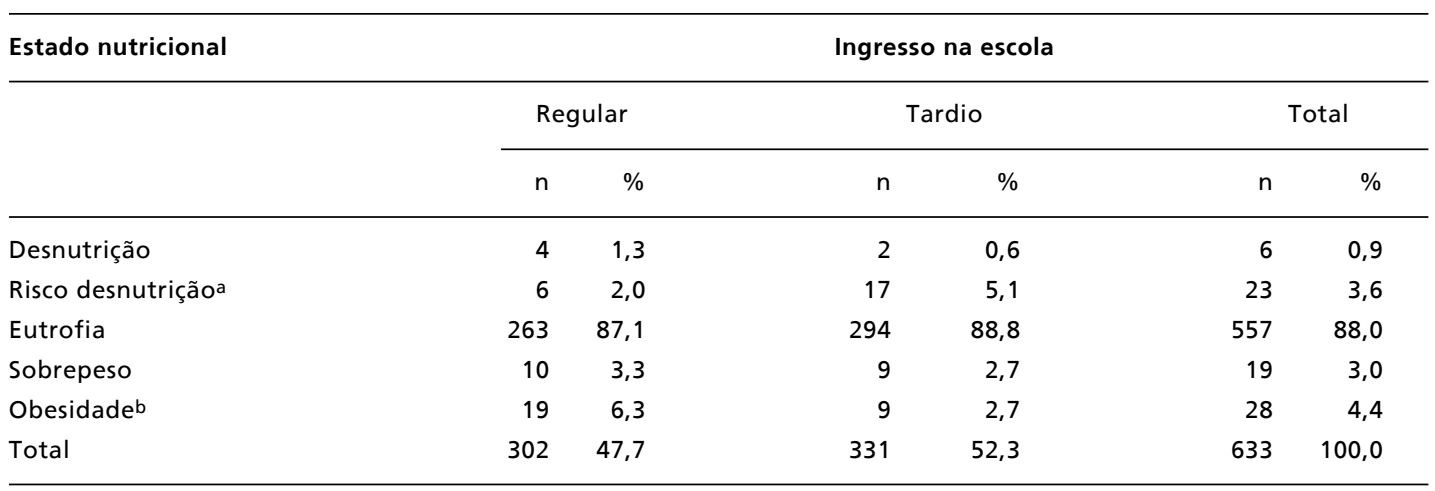

a $\chi^{2}=4,47 ;{ }^{*} p=0,034 ;$ b $\chi^{2}=4,77 ;{ }^{*} p=0,029$

de ingresso regular e 335 (52,6\%) ingressaram em idade tardia. Observou-se que não houve correlação entre a escolaridade materna, local de nascimento do escolar e a idade de ingresso da criança na escola. Foi estatisticamente significante a relação entre o sexo masculino e o ingresso tardio na escola.

Analisando o estado nutricional em relação ao sexo e ao local de nascimento não encontramos diferenças estatisticamente significantes. Na relação entre estado nutricional dos escolares e a escolaridade materna observamos significância estatística apenas na associação entre obesidade da criança e escolaridade materna $<4$ anos (Tabela 1 ).

Na Tabela 2 observamos significância estatística na relação de risco de desnutrição dos escolares e ingresso tardio na escola e de obesidade com ingresso regular. Nas demais categorias do estado nutricional não houve associação.

A análise do escore $z$ de E/I mostrou prevalência de baixa estatura em 7,8\% das crianças (IC95\%: 5,910,3 ), incluindo $0,5 \%$ das crianças com $z$ escore 3,0 . Em risco de baixa estatura estavam $8,8 \%$ dos escolares e $83,3 \%$ com estatura normal. Das 50 crianças $(7,8 \%)$ com baixa estatura, a maioria era do sexo masculino, no entanto não houve diferença estatisticamente significante. Observou-se também que não houve significância estatística quanto ao sexo das crianças e o risco de baixa estatura e estatura normal. Analisando a estatura dos escolares em relação à naturalidade, observou-se que o percentual de baixa 


\section{Figura 1}

Distribuição dos escolares de primeira série de escolas públicas estaduais, segundo a escolaridade materna e a estatura. Belém, Pará, 2001

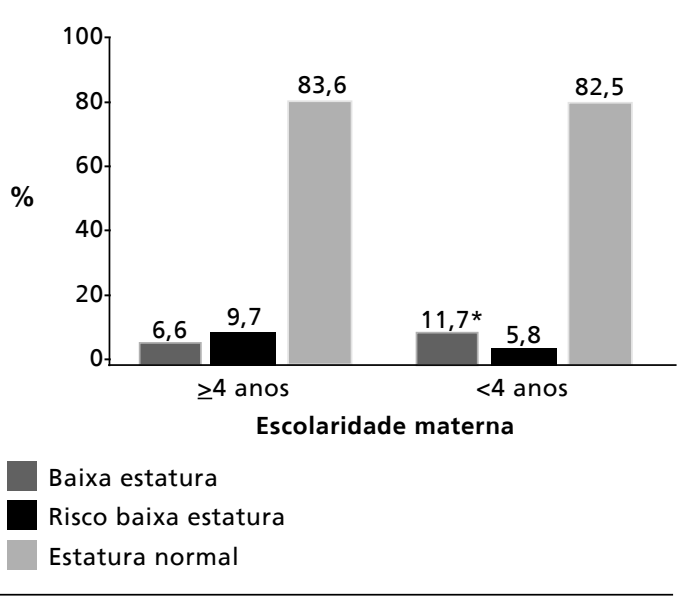

Baixa estatura $x<4$ anos; $p=0,041$ *

\section{Figura 2}

Distribuição dos escolares segundo a idade de ingresso na escola e a estatura.

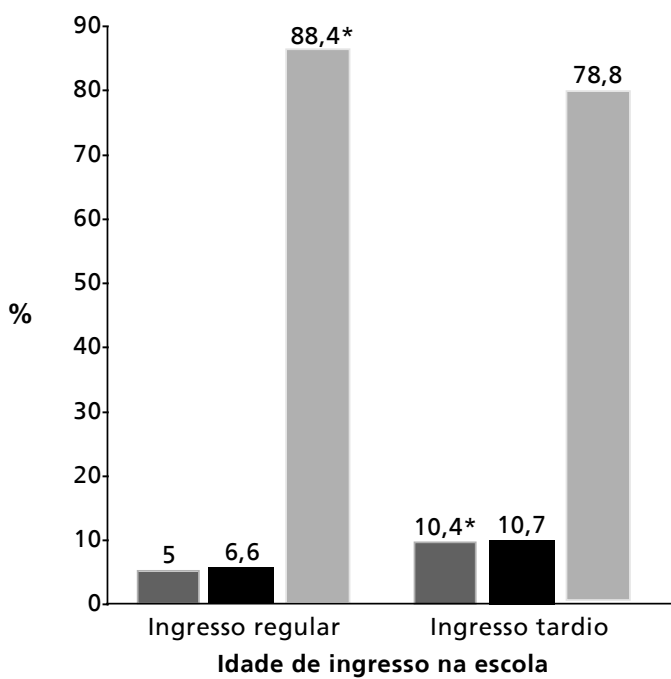

Baixa estatura

Risco baixa estatura

Estatura normal

Estatura normal $\mathrm{x}$ ingresso regular; ${ }^{\star} p=0,001$; baixa estatura $\mathrm{x}$ ingresso tardio; ${ }^{*} p=0,010$ estatura foi maior $(11,8 \%)$ nas crianças naturais de outros municípios e estados do que nas oriundas de Belém (7,3\%), mas não houve significância estatística. Também não houve significância estatística no risco para baixa estatura e estatura normal. $\mathrm{Na}$ associação entre anos de escolaridade materna e estatura dos escolares, observou-se percentual significantemente maior $(11,7 \%)$ de baixa estatura nas crianças cujas mães possuiam escolaridade menor que quatro anos (Figura 1).

Na relação da estatura com a variável idade de ingresso na escola observou-se que, das crianças com baixa estatura, a maioria ingressou na escola em idade tardia, sendo essa relação estatisticamente significante assim como o ingresso regular na escola dos escolares com estatura normal (Figura 2).

\section{Discussão}

Este é o primeiro inquérito antropométrico entre escolares de primeira série do ensino elementar, realizado na Região Norte do Brasil, que atua como instrumento de vigilância nutricional. As prevalências de sobrepeso e obesidade, da ordem de $7,4 \%$, quando consideradas conjuntamente, apontam para o crescimento desse desvio nutricional mesmo entre o subgrupo de menor renda de uma das regiões menos desenvolvidas do país.

Concomitantemente, identificaram-se taxas de baixa estatura de $7,8 \%$, mostrando que a desnutrição primária se mantém como problema prevalente mesmo entre os sobreviventes que se matriculam no primeiro ano da escola. Se considerássemos os não matriculados e os vieses de sobrevivência, tais taxas se aproximariam de preocupantes $20 \% .11$

Em Belém, não foram encontradas deficiências nutricionais importantes na população estudada, a qual era constituída por crianças que freqüentavam as escolas públicas estaduais.

No presente estudo foi estatisticamente significante a associação entre o sexo masculino e o ingresso escolar tardio. Algumas questões podem ser levantadas para explicar essa associação. Será que esses meninos estão sendo mão-de-obra para ajudar na renda familiar e, por essa razão, atrasam seu ingresso na escola? Ou ainda, será que, pelas condições de pobreza e falta de informação das famílias, a escolaridade não é valorizada, já que a grande luta é pela sobrevivência diária? Nesse último caso, por que as meninas teriam ingresso escolar mais precoce do que os meninos? Para esclarecer melhor tais questões é necessário que se conheçam dados como renda familiar, taxa de absen- 
teísmo e evasão escolar na cidade de Belém.

Foi observado nesta pesquisa que as mães de crianças oriundas de outros municípios e estados tinham menor escolaridade. Também essas crianças apresentaram maior percentual de ingresso escolar tardio, embora não significante, mas com certa tendência estatística. A escolaridade materna e a naturalidade seriam fatores que se potencializam, com uma interação sinérgica de migração e más condições de vida. A baixa escolaridade materna e o ingresso escolar tardio da criança confirmam as menores oportunidades de vida do migrante, com menor acesso à educação, à saúde e condições básicas de vida, já se iniciando na infância.

A prevalência de déficit de peso para estatura foi baixa $(0,9 \%)$, sendo que nenhuma criança estava abaixo de menos três escores z. No Brasil, em inquéritos nutricionais nacionais, observou-se que, em menores de cinco anos de idade, a prevalência de desnutrição em 1989 era de 2,1\% e em 1996 era de 2,3\%. Em 1996, a prevalência na região Norte foi de $1,2 \% .12$

Observou-se que 3,0\% dos escolares encontravam-se na faixa de sobrepeso e $4,4 \%$ na faixa de obesidade. A presença de percentual baixo de déficit nutricional e o aumento de obesidade da população de estudo sugerem a possibilidade de transição nutricional e a necessidade de atividades de vigilância nutricional. No último inquérito antropométrico nacional, a prevalência de obesidade entre os menores de cinco anos no Brasil foi de $4,9 \%$, e na região Norte foi de 4,5\%.13 O aumento da prevalência de obesidade no Brasil, em diferentes áreas e segmentos sociais, reflete a tendência de transição nutricional observada na comparação dos dois últimos inquéritos antropométricos nacionais. Ocorreram mudanças no sentido de maiores prevalências de obesidade nas regiões menos desenvolvidas do país (Norte e Nordeste) e reduções nas prevalências das mais desenvolvidas (Sul e Sudeste). Os aumentos e reduções nas respectivas regiões foram mais intensos em filhos cujas mães tinham maior escolaridade ( $>4$ anos) e entre menores de dois anos de idade. 12

No presente estudo houve associação estatisticamente significante entre obesidade e escolaridade materna menor que quatro anos, demonstrando, talvez, que já houve mudança na fase de transição nutricional da região Norte. Há o predomínio do desvio nutricional ainda em camadas com menor escolaridade devido ao desconhecimento da sua morbidade.

A obesidade na infância é um problema de saúde pública e as pesquisas demonstram associação entre esse evento e alta prevalência de hipertensão, diabetes, doenças respiratórias e ortopédicas e desordens psico-sociais. ${ }^{14}$ Uma epidemia global de obesidade está emergindo, tanto nos países desenvolvidos como naqueles em desenvolvimento, em crianças, adolescentes e adultos, que estão sendo seriamente afetados. Em muitos países a obesidade coexiste com a desnutrição. A obesidade é relativamente incomum na África e Ásia e é mais prevalente em populações urbanas que nas rurais, mas o aumento da prevalência pode ser mais alto nos países industrializados. As mudanças na alimentação e estilo de vida, resultantes da industrialização, urbanização, desenvolvimento econômico e globalização estão tendo impacto significante no estado nutricional das populações. 15

Em relação ao escore $z$ de estatura para idade observou-se, neste trabalho, que a prevalência de baixa estatura foi de 7,8\%, incluindo $0,5 \%$ abaixo de três escores $z$. A prevalência do risco de baixa estatura foi de $8,8 \%$. Comparando os resultados desta pesquisa com outras realizadas no Brasil com dados de antropometria de escolares ao ingresso no ensino fundamental, observou-se que a prevalência da baixa estatura é muito variada, demonstrando o desenvolvimento socioeconômico da região em que foi realizado. Na cidade de Florianópolis, em Santa Catarina, o censo foi descaracterizado por algumas escolas que não permitiram a realização da pesquisa e o resultado da investigação revelou uma prevalência de 3,1\% de déficit de estatura. 5 Em Osasco, São Paulo, no censo do município, foi encontrada uma prevalência de 4,8\%.6 No Ceará, Paraíba e Piauí foram realizados censos estaduais de estatura para a idade. No estado da Paraíba, foi encontrada uma prevalência de $14,5 \%$ para todo o estado, com um déficit de $17,86 \%$ de estatura na zona rural e de $11,87 \%$ na zona urbana. ${ }^{7}$ No estado do Ceará, a prevalência encontrada de déficit de estatura foi de $17,2 \% 18$ e no estado do Piauí, a prevalência foi de $28 \%$.

Quanto à relação entre sexo e baixa estatura, em Belém, houve predomínio no sexo masculino, embora sem significância estatística, estando de acordo com os resultados apresentados em outras pesquisas. No Ceará, os escolares do sexo masculino apresentaram percentuais mais elevados de baixa estatura, sendo que na zona urbana a taxa foi de $17,61 \%$ e na zona rural foi de $23,17 \%$; para o sexo feminino os percentuais na zona urbana foram de $12,74 \%$ e na zona rural foram de $17,41 \% .16$ No Piauí, para o sexo masculino na zona urbana, o déficit de estatura foi de $24,1 \%$, de $36,6 \%$ na zona rural e $32,1 \%$ para todo o estado; para o sexo femi- 
nino foi de $17,0 \%$ na zona urbana, de $26,9 \%$ na zona rural e de $23,2 \%$ para todo o estado. 17

No inquérito antropométrico brasileiro de 1996, em crianças menores de cinco anos de idade, a prevalência de baixa estatura foi de $10,5 \%$, e na região Norte, foi de $16,2 \% .10$ A prevalência estimada de baixa estatura na América do Sul diminuiu de $25,1 \%$, em 1980 , para $9,3 \%$ em 2000 , e é previsto que, em 2005, seja de 5,3\%, segundo dados da Organização Mundial de Saúde, após a análise de dados de pesquisas representativas de 241 países desde 1980.14 A América Latina e o Caribe têm índices mais baixos de retardo estatural em pré-escolares do que a África e Ásia e do que a média global dos países em desenvolvimento. 18

Em nossa pesquisa houve associação estatisticamente significante entre a baixa estatura e a escolaridade materna menor que quatro anos e o ingresso tardio na escola. Não houve influência das variáveis estudadas sobre o risco de baixa estatura. A associação entre a estatura normal e o ingresso regular na escola também mostrou significância estatística. Isso demonstra o sinergismo de condições inadequadas sobre a determinação do déficit de crescimento.

\section{Referências}

1. Habicht JP, Martorell R, Yarbrough CH, Malina R, Klein RE. Height and weight standards for preschool children. How relevant are ethnic differences in growth potential? Lancet 1974; 1: 611-4.

2. OPS (Organización Panamericana de la Salud), Fondo del as Naciones Unidas para la Infancia (UNICEF). Los censos de talla y sus usos: informe técnico, CT45. Washington (DC); 1997.

3. Valverde V, Delgado H, Flores R, Sibrián R, Palmieri M. Uses and constraints of schoolchildren's height data for planning purposes: national experiences in Central America. Food Nutr Bull 1985; 8: 42-48.

4. Monteiro CA. Coleta e análise da altura dos alunos ingressantes nas escolas do primeiro grau do país: uma proposta para um sistema nacional de acompanhamento do estado de saúde e nutrição da população. J Pediatr 1989; 65: 89-92.

5. Corso ACT, Caldeira GV, Buralli KO. Estatura de escolares ingressantes na rede básica de ensino do município de Florianópolis. Rev Cienc Saúde 1999; 18: 57-61.

6. Mondini L, Monteiro CA. A coleta da altura de alunos ingressantes nas escolas de primeiro grau em um sistema de vigilância nutricional: análise dos dados antropométricos. J Pediatr 1994; 70: 273-9.

7. Carvalho AT, Costa MJC, Ferreira LOC, Batista Filho M. Cartografia do retardo estatural em escolares do Estado da Paraíba, Brasil. Rev Saúde Pública 2000; 34: 3-8.
A necessidade da realização de estudos antropométricos se evidencia pela iniciativa da Organização Mundial de Saúde em manter o programa "WHO Global Database on Child Growth and Malnutrition", uma compilação padronizada de dados de crescimento de crianças abaixo de cinco anos de idade, baseada em estudos transversais em países da África, Ásia, América Latina e Oceania desde 1980.11 A informação dos censos de estatura na América Central e em outros países da América Latina, da Ásia e África tem permitido conhecer a situação nutricional do escolar como indicativo do grau de desenvolvimento humano da população. 19

A realização de censos nacionais como instrumento de vigilância nutricional seria importante no sentido de identificação dos riscos nutricionais e como indicador de políticas de saúde no Brasil, a exemplo do que já é realizado em outros países. $\mathrm{Na}$ vigência de uma situação mundial de transição nutricional, estudos periódicos utilizando os indicadores estatura para a idade e peso para estatura também devem ser incentivados.

8. WHO (World Health Organization). Physical status: the use and interpretation of anthropometry. Geneva: 1995. (Technical Report Series, 854).

9. Hamill PV, Drizd TA, Johnson CL, Reed RB, Roche AF. NCHS growth curves for children birth - 18 years. United States. Vital Health Stat 11 1977; (165):i-74.

10. Siegel S, Castellan NJ Jr. Non parametric statistics. New York: McGraw-Hill; 1988.

11. Oliveira O, Taddei JA. Efeito dos vieses de sobrevivência nas prevalências da desnutrição em crianças no sexto ano de vida. Brasil PNSN, 1989. Cad Saúde Pública 1988; 14: 493-9.

12. Taddei JA, Colugnati FA, Rodrigues EM, Sigulem DM, Lopez FA. Desvios nutricionais em menores de cinco anos. São Paulo: Universidade Federal de São Paulo; 2002.

13. Ministério da Saúde. Pesquisa Nacional sobre Demografia e Saúde 1996. Rio de Janeiro: BEMFAM; 1997.

14. de Onis M, Frongillo EA, Blössner M. Está disminuyendo la malnutrición? Análises de la evolución del nivel de malnutrición infantil desde 1980. Bol Org Mundial Salud 2001; 4: 100-10.

15. WHO (World Health Organization). Nutrition for health and development. A global agenda for combating malnutrition. Geneva; 2000. 
16. Fortaleza. Secretaria de Educação e Cultura. Fundação de Amparo ao Estudante. Primeiro censo estadual de altura/idade dos escolares de primeira série do ensino fundamental do Estado do Ceará. Fortaleza; 1993.

17. Piauí. Secretaria de Educação do Estado do Piauí. Secretaria de Educação e Cultura. Fundação de Amparo ao Estudante. Primeiro censo estadual de altura/idade dos escolares de primeira série do ensino fundamental do Estado do Piauí. Terezina; 1993.
18. Delgado HL. Nutrición y alimentación en América Latina y el Caribe: en pro de la seguridad alimentaría y nutricional. In: Encuentro Latinoamericano y del Caribe de Proyectos de Nutrición Humana; 2000 dic 4-8; San Salvador. (Publicación INCAP CE/118).

19. Fulladosa PP. Los censos de talla en escolares: consideraciones metodológicas. Guatemala: INCAP; 2000. (Notas Técnicas, 26).

Recebido em 20 de outubro de 2004

Versão final apresentada em 4 de janeiro de 2006

Aprovado em 24 de janeiro de 2006 